\title{
Externally Induced Rotation of the Resistive Wall Modes in RFX-mod
}

\author{
V. Igochine $^{1^{*}}$, T. Bolzonella ${ }^{2}$, S. C. Guo ${ }^{2}$, D. Yadikin ${ }^{1}$, M. Baruzzo ${ }^{2}$, H. Zohm ${ }^{1}$ \\ ${ }^{1}$ MPI für Plasmaphysik, Euratom-Association, D-85748 Garching, Germany \\ ${ }^{2}$ Consorzio RFX, Associazione Euratom-ENEA sulla fusione, Padova, Italy
}

\begin{abstract}
This paper reports on the active rotation experiments in RFX-mod where it is demonstrated for the first time in RFPs that an internal non-resonant resistive wall mode can be "unlocked" from the resistive wall using an external perturbation. The observed constant rotation of the mode depends on the phase shift between the external perturbation and the mode. It is seen that plasma rotation, plasma current and coupling to other modes have no impact on the rotation frequency of the mode. A simple analytical model is proposed which gives a good description of the experimental results.
\end{abstract}

\section{Introduction}

Resistive Wall Mode (RWM) instabilities are currently the main performance limiting MHD phenomena in reversed field pinch (RFP) devices - once the discharge duration exceeds the typical wall time of the passive magnetic boundary surrounding the plasma (such as the vacuum vessel and/or resistive shell) regardless of the operational scenario. Hence, in recent years significant effort has been applied to active control of RWMs in RFPs. For example, it was demonstrated that multiple static, non resonant, current driven RWMs can be completely suppressed by the feedback action of a set of active magnetic coils [1,2]. Active control techniques are of common interest for many toroidal magnetic confinement concepts. The RWM in a tokamak typically rotates with respect to the wall with a rotation frequency (in the lab. frame) which is much smaller than the plasma rotation frequency. Plasma rotation provide a drag force which rotates the RWM. Unfortunately, plasma rotation though it is expected to be strongly reduced in future large devices such as ITER. The role of plasma rotation as a stabilizing mechanism is one of the main differences between tokamaks and RFPs. In RFP plasmas, RWMs are usually observed as non-resonant, wall locked instabilities, and experience little influence on their growth rates from either plasma fluid rotation, error field minimization, or the presence of other MHD instabilities such as tearing modes. Here, only remaining stabilization strategy is the use of an active coil system, coupled

\footnotetext{
${ }^{*}$ E-mail: Valentin.Igochine@ipp.mpg.de
} 
to a set of magnetic sensors by a feedback controller. Depending on the flexibility of the controller implemented, control strategies can vary from the simple implementation of the Virtual Shell idea, originally introduced by Bishop in 1989 [3], where the active coil action is aimed at canceling the measurement of a set of radial field sensors, to more sophisticated controls with non-zero references or full complex gains in the feedback circuit. This work is focused on the influence of the external perturbations on the resistive wall mode in RFP. It continues and extends the recent letter [4] and conference contribution [5]. Section II reports the experimental results from static perturbation experiments, open-loop rotation experiments and closed-loop rotation experiments. A simple cylindrical model is discussed in section III. Finally, section IV discusses the implications of these results for further studies of the RWMs.

\section{Results of the rotation experiments on RFX}

\section{II a. Control system}

The RFX-mod RFP device (Padova, Italy) has thin copper shell with a vertical field penetration time of $\tau_{V \text {,shell }}=50 \mathrm{~ms}$. RFX-mod is equipped with a flexible active system for MHD mode control [6]. It consists of 192 active coils, fed by 192 independent amplifiers. The active coil set entirely covers the RFX-mod external surface, providing control on the radial component of the perturbed magnetic field. A digital PID controller allows various control schemes to be applied. A schematic of the active control system is shown in figure 1 . Signals from a large number of sensors measuring all three components of the perturbed magnetic field provide the input for the control system. The signals are decomposed with an FFT algorithm and converted (according to the chosen control scenario) by a digital controller into "reference" signals corresponding to the magnetic field of each harmonic. An inverse FFT transform then produces the control currents for each saddle coil via individual coil power supplies. Maximum latency time of the control loop is $330 \mu \mathrm{s}$. 


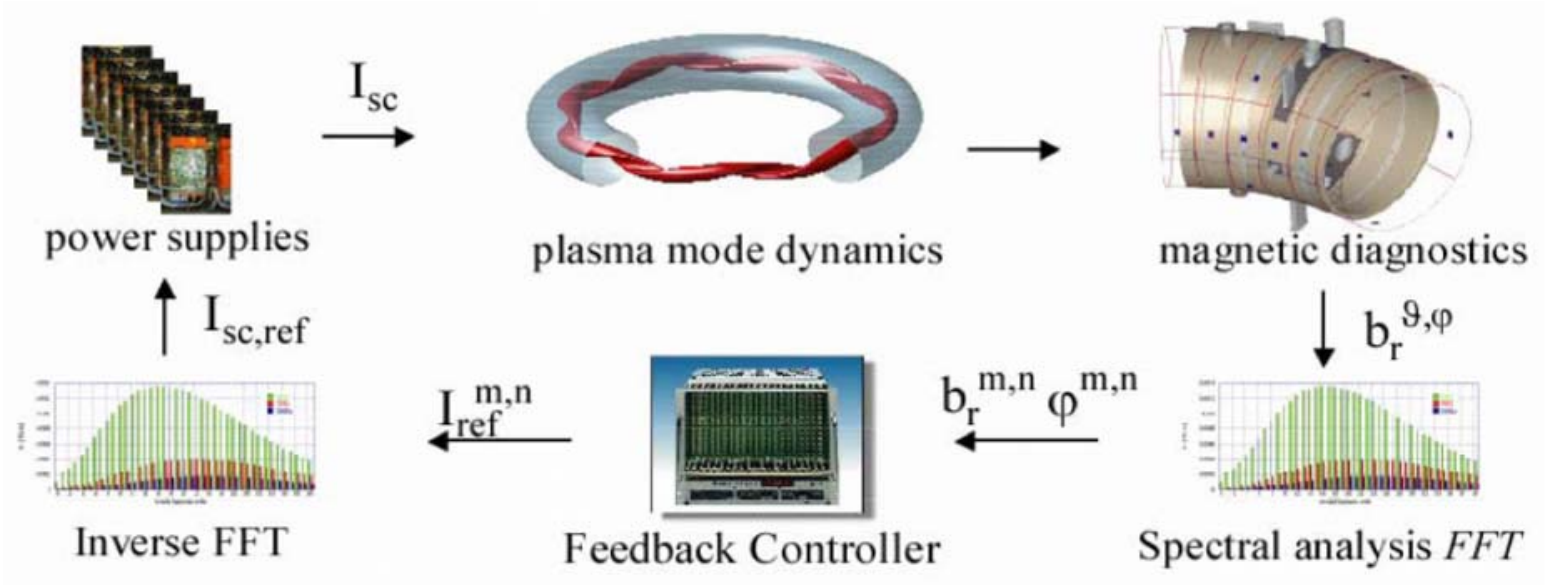

Figure 1. Schematic illustration of the active control system.

Using this system it is possible to control any particular mode with chosen poloidal (m) and toroidal (n) mode numbers. This technique has been widely used in RFPs to investigate the dynamics of the mode of interest separately from other MHD activity. In the reported experiments, the target mode for investigation was the most unstable internal non-resonant resistive wall mode, i.e. an instability with toroidal mode number $\mathrm{m}=-6$ and poloidal mode number $n=1$. Other modes were suppressed by active control in order to optimize the plasma discharge. Note that total field measured by the sensors $b_{r}$ (fig.1 ) includes both the plasma and external parts. A model was used [7] in order to separate these parts in the total measured signal. In the following we denote the plasma part with the subscript 'pl', the external part with 'ext' and the total field with 'tot'.

\section{II b. Static perturbations and open-loop experiments}

We start our investigations by applying static external perturbations. Time traces of the amplitude and phase of the plasma and external perturbations are shown in fig. 2. Two cases are compared: (i) the mode is growing freely until $0.1 \mathrm{~s}$ and then controlled after this time using feedback ( $B_{p l}$ : free growth); (ii) the mode is growing freely until $0.05 \mathrm{~s}$ and then a static external perturbation is applied ( $B_{p l}:$ static field). The amplitude of the external static field is also shown for the second case ( $B_{\text {ext }}$ : static field). After the start of the external static perturbation the mode reappears at different toroidal position so that its phase coincides with the phase of the external perturbation and so the mode begins to grow. 

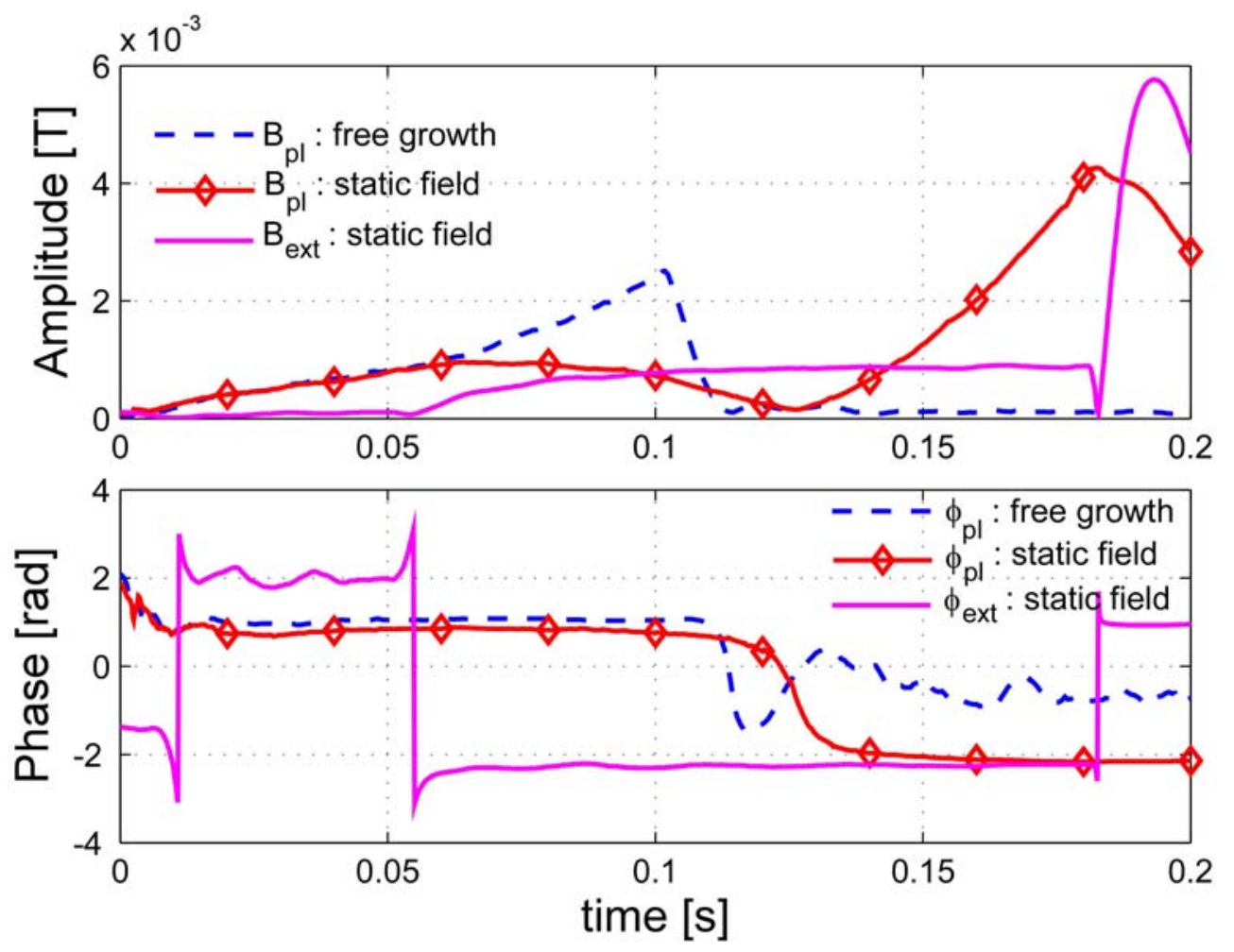

Figure 2. Results of the experiments for free growing mode and static perturbation. Dashed line - free growing mode till $0.1 \mathrm{~s}$ and controlled after this time using feedback $\left(B_{p l}, \phi_{p l}\right.$ : free growth); solid line with diamonds - plasma mode with applied static perturbation from $0.05\left(B_{p l}, \phi_{p l}\right.$ : static field); solid line - external perturbation ( $B_{e x t}, \phi_{e x t}$ : static field). In this figure, the plasma part of the signals is separated from the external perturbation amplitude from the coils.

The next set of experiments were made with rotation of external perturbations in openloop. An external perturbation was applied with different amplitudes and rotation frequences $(\mathrm{f}=1-30 \mathrm{~Hz}$ ). Time traces for a discharge with $\mathrm{f}=2 \mathrm{~Hz}$ is shown in figure 3 . After the switchon of the external rotating field a change in the phase of the mode $\phi_{p l}$ is observed. This indicates a short period where the mode rotates slowly (between $0.1 \mathrm{~s}$ and $0.13 \mathrm{~s}$ ) accompanied by relatively constant amplitude of the mode $B_{p l}$. However, the relative balance between the mode-to-wall and the mode-to-external perturbation interactions is too small (i.e. the wall dominates over the external perturbation) which leads to complete wall locking at about $\mathrm{t}=0.14 \mathrm{~s}$. From this point on, the mode displays a constant phase (locked to the wall) and growing amplitude. The main problem of the open-loop rotation technique is the nondomination of the "wall-external" field interaction. This problem can be solved using a closed-loop technique as shown below. 


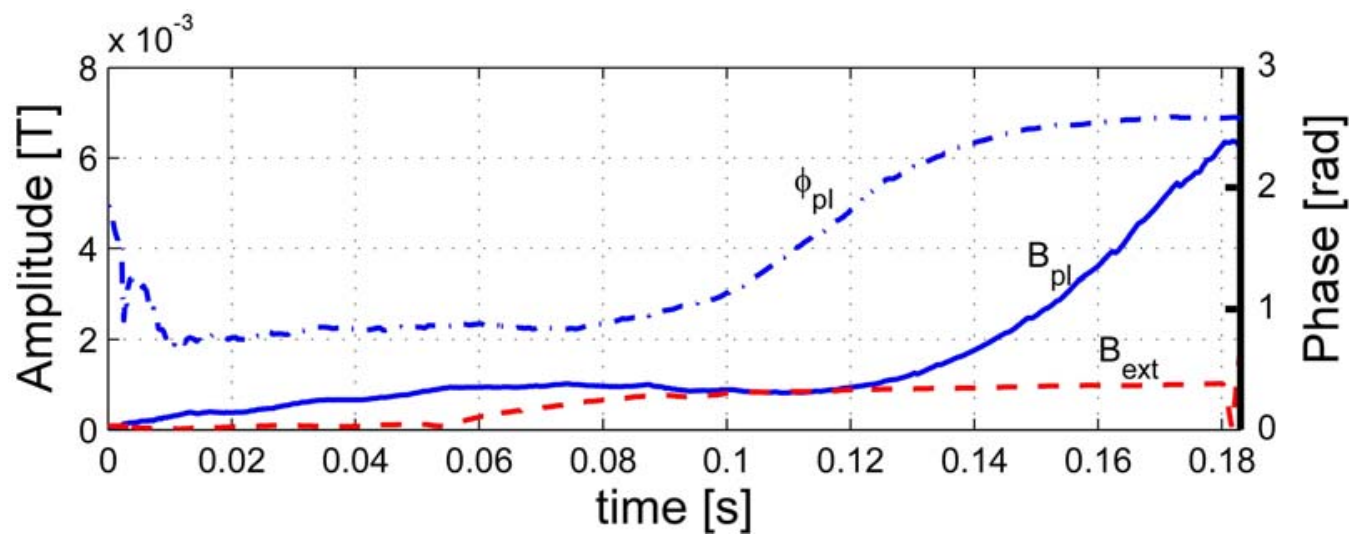

Figure 3. Open-loop rotation experiments. Solid line - plasma mode amplitude $B_{p l}$; dashed-doted line phase of the plasma mode $\phi_{p l}$; dashed line - external perturbation amplitude $B_{e x t}$ (rotation frequency: $\mathbf{f}=\mathbf{2 H z})$.

\section{II c. Closed-loop experiments}

The final set of experiments were made with a closed-loop circuit. The principle schemes of the feedback action are shown in figure 4.

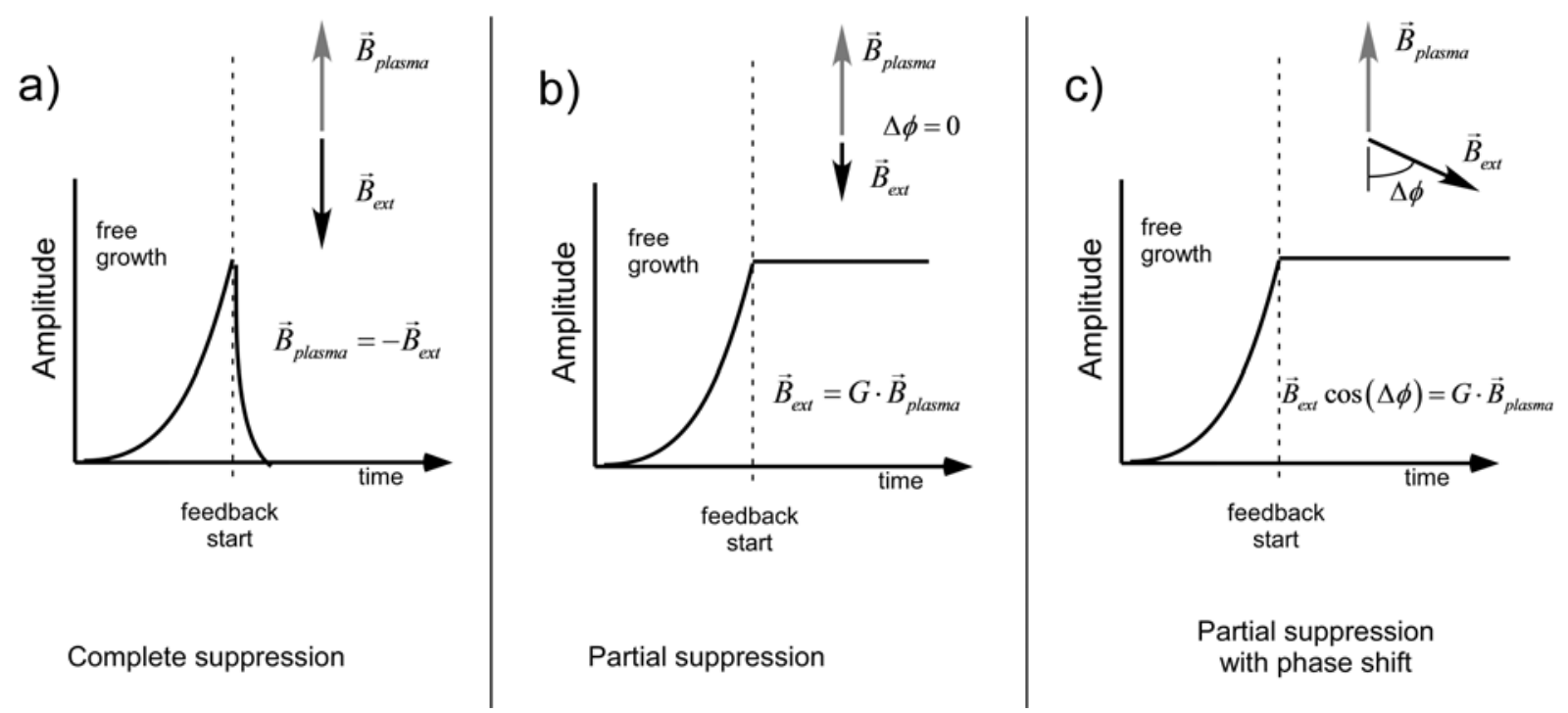

Figure 4. Principle schemes of the closed-loop feedback experiments and their influence on the mode amplitude. a) The mode amplitude is suppressed completely after start of the feedback. $\vec{B}_{p l}=-\vec{B}_{e x t}$; b) Partial suppression of the mode amplitude. Feedback keeps the mode amplitude at a constant level, $\Delta \phi=0$, starting point for our experiments; c) Partial suppression with phase shift. Feedback keeps the 
mode amplitude at a constant level and rotate it $(\Delta \phi \neq 0)$; Note that $\left|B_{\text {ext }}\right| \cos (\Delta \phi)$ is the same for (b) and (c).

Full suppression of the mode amplitude is shown on fig 4a. Here, the plasma mode is cancelled by the feedback field resulting in the mode amplitude going to zero. Partial suppression is obtained with a smaller $B_{\text {ext }}$ (reference case $\Delta \phi=0$ ) which keeps the mode amplitude at a constant level (figure 4b). In this case, "partial suppression" means the growth rate is forced to zero, but the mode amplitude has a constant "finite" value. This allows one to investigate the influence of phase shifted external perturbations, as shown below. For cases (a) and (b), the imaginary part of the gain is zero $(\Delta \phi=0)$. In fig $4 \mathrm{c}$ partial suppression of the mode amplitude is obtained by applying a complex gain with a non-zero imaginary part. This corresponds to introducing an angle $(\Delta \phi \neq 0)$ between the plasma and external components. Note that this angle is kept constant during feedback which is different from the open-loop rotation experiment where $\Delta \phi$ varies with time. In the experiments with complex gain the projection of externally applied magnetic field $\left(B_{\text {ext }}\right)$ on the direction of the plasma field $\left(B_{p l}\right)$ is a constant $\left(\left|B_{e x t}\right| \cos (\Delta \varphi)=\right.$ const $)$ and has the same value for figures $4 \mathrm{~b}$ and $4 \mathrm{c}$.

The last set of experiments were made in closed-loop (feedback) at low (400kA) and high $(600 \mathrm{kA})$ plasma currents. It was found in both cases that application of complex gain $(\Delta \phi \neq 0)$ in the closed-loop operation was able to rotate the plasma mode, as clearly seen in figure 5. Here, the plasma perturbations are plotted separate from the total signal, which include also perturbations from the coils. 

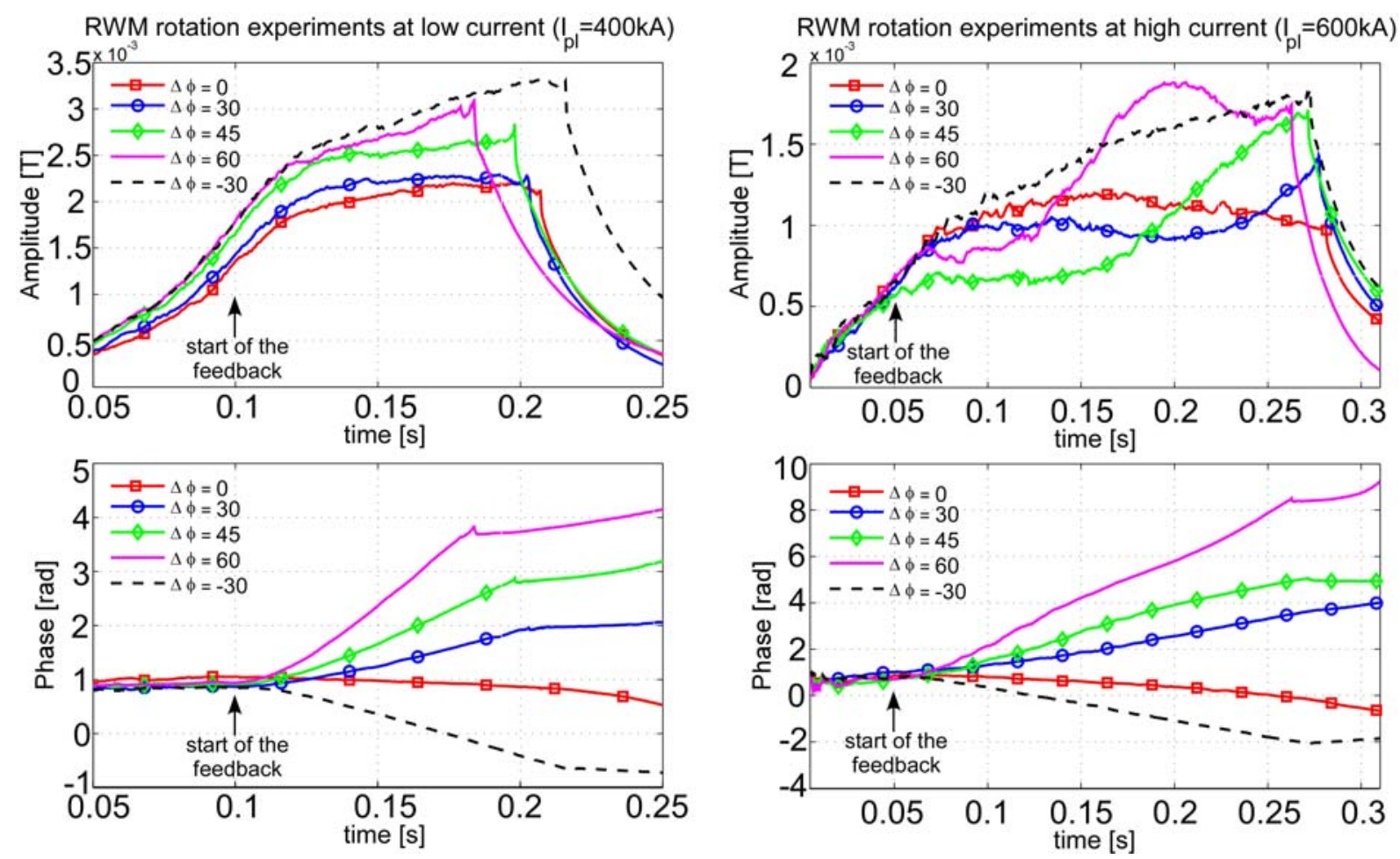

Figure 5. Results of the RWM rotation experiments with closed-loop feedback at two different plasma currents. The The amplitudes $B_{p l}$ and phases $\phi_{p l}$ are shown for $(\mathrm{m}=\mathbf{1 , n}=-6)$ mode. $\Delta \phi$ is angle between the plasma and external perturbation.

In the following the reference case for partial suppression of the mode amplitude corresponds to figure $4 \mathrm{~b}$ with $\Delta \phi=0$. For this case the $(1,-6)$ unstable RWM grows freely for the first $100 \mathrm{~ms}$ (400kA plasma current) or 50ms (600 kA plasma current). After which, a small real gain was applied to keep the mode amplitude at a finite value, without complete suppression. Then complex gain with different values of imaginary part were applied, as discussed above. In some of the high current discharges the mode varied slightly in amplitude, phase changes nevertheless, remain the same in spite of changes in the mode amplitude. Thus, the rotation frequency also remains constant even with increasing mode amplitude. The main RFP equilibrium characteristics: field reverse ratio $\left(F=B_{z}(a) /\left\langle B_{z}\right\rangle\right)$ and pitch parameter $\left(\Theta=B_{\theta}(a) /\left\langle B_{z}\right\rangle\right)$ also remain constant during the mode amplitude growth phase. The reason for this behaviour is not fully understood and will be investigated in future experiments. 


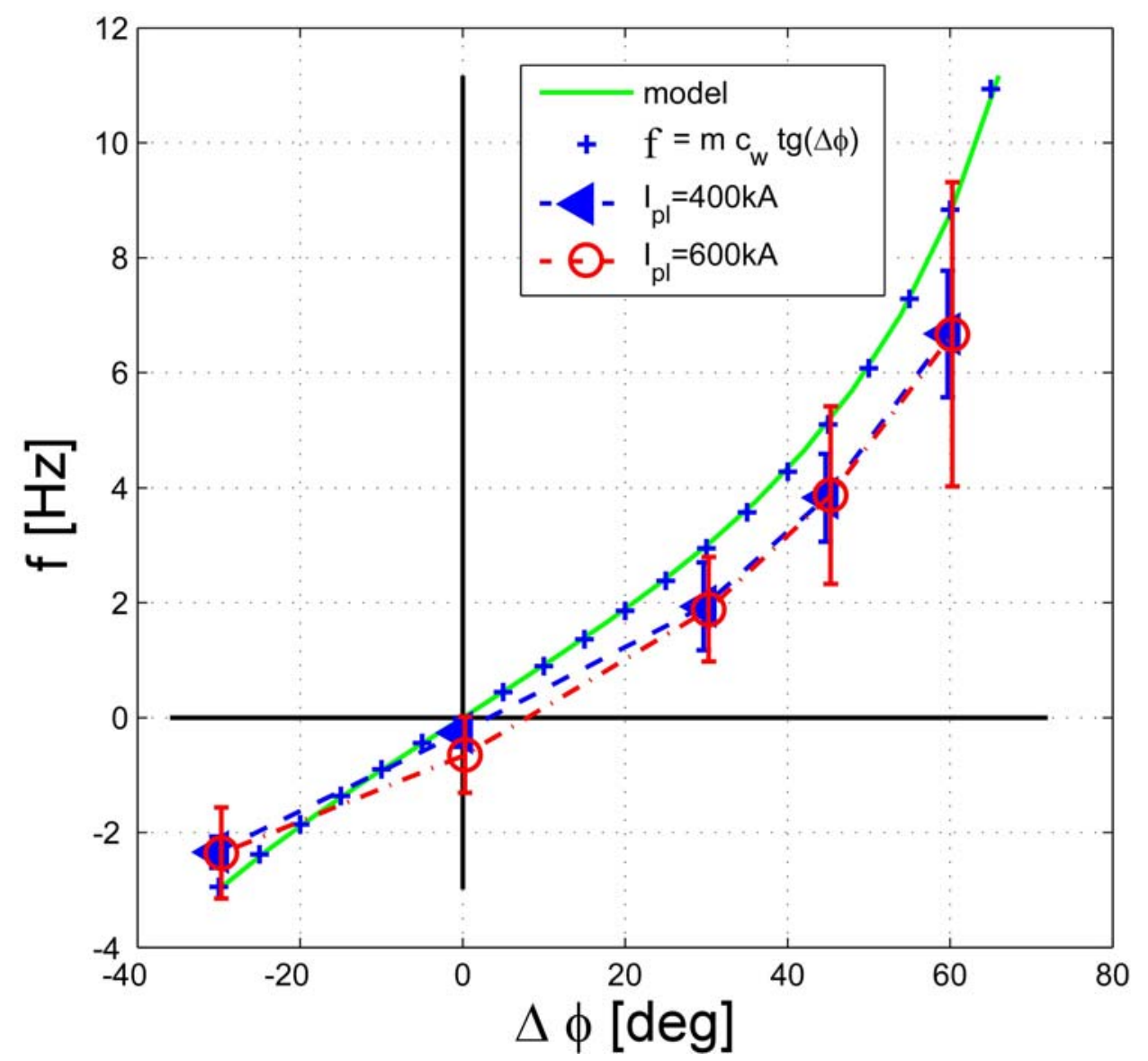

Figure 6. Dependence of the mode rotation frequency on the phase shift $\Delta \phi$ between the mode and external perturbations. Phase information from the phase traces presented in fig.5 is summarized in this figure. The models are discussed in part III.

In order to estimate the effect of varying imaginary gains, the phase information from the experimental traces presented in figure 5 are collected and presented in figure 6 . The resulting frequency values are shown in the figure with triangles $\left(\mathrm{I}_{\mathrm{pl}}=400 \mathrm{kA}\right)$ and open circles $\left(I_{\mathrm{pl}}=600 \mathrm{kA}\right)$. The error bars shown represent the time averaging of the frequency obtained from the experimental results over the time interval of the constant rotation (see fig. 5). The magnitude of the error bar is given by the standard deviation which depends on the length of the averaging interval. The feedback system keeps the phase shift between the mode and externally applied perturbation at a constant value during the feedback. Experimental results show that the rotation frequency of the mode strongly depends on the phase shift and has no dependency on plasma current. Plasma rotation at low frequencies is also not important which is demonstrated by applying the rotated perturbations in two opposite directions $(\Delta \phi= \pm 30)$. Equivalence of the results at both currents can be also seen in figure 7. Here, the evolution of the mode rotation frequency are shown directly after the feedback switch-on $(\mathrm{t}=0.0 \mathrm{~s}$ in figure 
7). One can see that not only stationary frequencies $\left(t>\tau_{\text {wall }}\right)$ are the same as shown in figure 6 , but also the initial change of the rotation frequencies $\left(t<\tau_{\text {wall }}\right)$ is identical within experimental errors. The time required for the frequency to grow to a stationary value is about the same for all curves and corresponds to the resistive wall time. This indicates that the wall properties and penetration of the perturbation determine the mode acceleration.

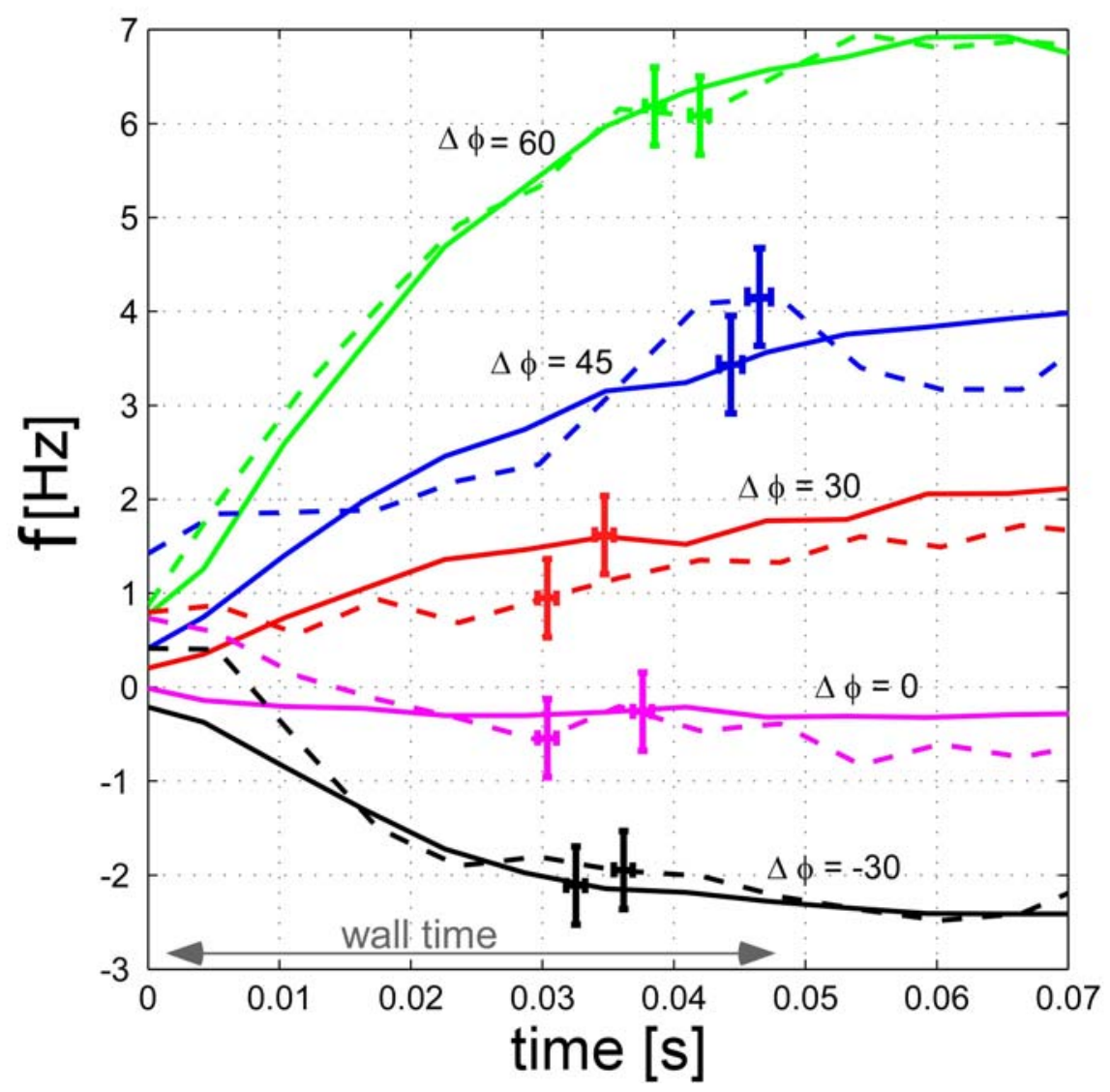

Figure 7. Rotation frequency after beginning of the feedback for 400kA (solid lines) and 600kA (dashed lines). Time point zero corresponds to the start of the feedback. Wall time is indicated. 


\section{Modelling of the rotation experiments}

For the analysis of the mode acceleration a periodic cylindrical model is proposed. Similar models were used previously for the investigation of the feedback behaviour $[8,9$, 10]. The model assumes a plasma of radius $r_{a}$ surrounded by a resistive wall at $r_{b}$ and the feedback coil at $r=r_{f}$. The indexes $b$ and $f$ correspond to the resistive wall and the feedback coils respectively. In the vacuum region outside the plasma, the most general solution of the Laplace equation $\Delta \Psi=0$, with $\vec{b}=\nabla \Psi$ ( $\overrightarrow{\mathrm{b}}$ is the magnetic fluctuation field) and the solution for the radial part of perturbed magnetic flux can be expressed as $\Psi(r)=A_{j} k r I_{m}^{\prime}(k r)+B_{j} k r K_{m}^{\prime}(k r)$, where $I_{\mathrm{m}}(k r)$ and $K_{\mathrm{m}}(k r)$ are the modified Bessel functions, $k=n / R$ and $j$ is the index for the vacuum region. Considering only one external kink mode with poloidal mode number $m$ and toroidal mode number $n$, the perturbed magnetic flux is written as

$$
\Psi_{m, n}(r, \theta, \phi, t)=\Psi_{m, n}(r) \cdot \exp (i \cdot(m \theta-n \varphi-\omega t))
$$

Using a similar approach as Ref. [11], the total magnetic flux is expressed as the linear combination of plasma and coils parts

Applying Ampere's law at $r_{b}$ and $r_{f}$, and asymptotic matching the above solutions in vacuum region, the following relations at the resistive wall and at the coils are obtained

$$
\begin{aligned}
& {\left[r \frac{d \Psi_{m, n}}{d r}\right]_{r_{b}^{-}}^{r_{b}^{+}}=E_{b, b} \Psi_{b}+E_{b, f} \Psi_{f}=-i \cdot \omega \cdot \tau_{b} \Psi_{b} } \\
\text { and } \quad & {\left[r \frac{d \Psi_{m, n}}{d r}\right]_{r_{f}^{-}}^{r_{f}^{+}}=E_{f, b} \Psi_{b}+E_{f, f} \Psi_{f}=-\mu_{0} S(m, n) I_{f}\left(m^{2}+n^{2} \varepsilon_{f}^{2}\right) }
\end{aligned}
$$

where $E_{i, j}=\left[r \frac{d \hat{\psi}_{j}(r)}{d r}\right]_{r_{i}^{-}}^{r_{i}^{+}} i, j=b, f$;

and $\tau_{b}=\mu_{o} \sigma \delta r_{b}, \omega=\omega_{r}+i \gamma . \mathrm{S}(\mathrm{m}, \mathrm{n})$ is a coefficient related to the structure of the feedback coils. For the feedback circuit a simple model equation is adopted:

$$
-L_{f} \frac{d I_{f}}{d t}-\left(\Psi_{b} G\right)=R_{f} I_{f}
$$


where the sensors are located at $r_{b} . I_{f}$ is the current in the feedback coils, $L_{f}$ its inductance and $R_{f}$ its resistance. The final dispersion relation has the following form:

$$
\begin{gathered}
\hat{E}_{b, b}+i \cdot \omega \cdot \tau_{b}+\frac{E_{b, f}}{E_{f, f}} \frac{\hat{G}}{1-i \cdot \omega \cdot \tau_{f}}=0 \\
\text { where } \hat{E}_{b, b}=E_{b, b}-\frac{E_{f, b} E_{b, f}}{E_{f, f}}, \hat{G}=\frac{\mu_{0} S G}{R_{f}}\left(m^{2}+n^{2} \varepsilon_{f}^{2}\right), \tau_{f}=L_{f} / R_{f}
\end{gathered}
$$

Here, the gain $(G)$ is a complex quantity. The feedback system keeps the phase shift constant $(\Delta \phi=\operatorname{arctg}(\operatorname{Im}(G) / \operatorname{Re}(G))=$ const. $)$. A linear stability code is used to solve the Newcomb's equation for the non-resonant internal kink mode and to calculate the instability index $\hat{E}_{b, b}$ for the $(1,6) \operatorname{RWM}\left(F=-0.05, \Theta=1.47, \alpha=3.0, \varepsilon_{0}=0.23\right)$. Without feedback, the growth rate can be obtained from $\gamma \tau_{b}=\hat{E}_{b, b}$. The other indexes $E_{i j}$ have been calculated by asymptotically matching the vacuum solutions of the modified Bessel functions. The feedback coil $\mathrm{L} / \mathrm{R}$ time constant is taken as $\tau_{f} \approx 2 m s$. In the computation, the experiment is simulated using the following steps: first the value of $\operatorname{Re}(\hat{G})$ is found such that it keeps the $(1,-6)$ mode growth rate almost zero with $\Delta \phi=0$ (without rotation); then by keeping the same $\operatorname{Re}(\hat{G})$ and varying $\Delta \phi$ the corresponding complex $\omega$ is obtained from the dispersion relation, which gives the mode rotation frequency. It is found that the dispersion relation predicts the experimentally observed dependence of mode rotation frequency on the phase shift very well, as shown in figure 6 (solid line). The problem can be solved with further simplifications: no plasma response; ideal feedback; a more simple ansatz for the perturbed flux $\left(\Psi(r) \sim r^{ \pm m}\right)$; close position of the plasma boundary, resistive wall and feedback coils. These assumptions reduce the final result to a much simple expression:

$$
f \approx m \cdot c_{w} \cdot \operatorname{tg}(\Delta \phi) \approx 5.1 \cdot \operatorname{tg}(\Delta \phi)
$$

where $m$ is the poloidal mode number and $c_{w}=1 / \mu_{0} \sigma \delta$ is the wall parameter which depends only on its conductivity $\sigma$ and wall thickness $\delta$. This solution also agrees well with the previous more general model (see figure 6). This suggests that in our experiments:

- The rotation frequency of the plasma mode is defined by the frequency of the system: external coils + resistive wall + sensor. Such result indicates that the ideal mode has 
no interaction with bulk plasma flow in our experiments (i.e. no inertia and no dissipation). Thus, rotation frequency is defined by the wall properties and $\Delta \phi$.

- The time delay due to the feedback is not important as it is seen from similarities between general and simplified models. Thus, the "ideal feedback" assumption is valid for the presented results.

The equation (7) gives the infinite rotation frequency at $\Delta \phi=90$. It is also seen from figure $4 c$ that such an angle requires infinite gain G. It is clear that this is not achievable. Further experiments are therefore necessary to clarify the maximum possible frequency. One should note that such experiments may give also an estimate of the plasma rotation influence on the resistive wall mode. The presence of influence of the plasma rotation will appear as an asymmetry between co- and counter-rotation cases in figure 6 . In fact, the plasma rotation is the only asymmetry which is present in the experiment. The control system produces identical fields in co- and counter direction. In the asymmetric case the proposed simplified model would not be applicable because inertia and dissipation must be taken into account. Thus, further experiments with higher rotation in both directions would clarify how "ideal" is our resistive wall mode.

\section{Conclusions and Discussion}

It has been demonstrated for the first time in RFPs that the RWM can be unlocked from the resistive wall using an external perturbation. The observed constant rotation of the mode is slower than the inverse resistive wall time and depends on the phase shift $\Delta \phi$ between the external perturbation and the plasma mode. It was found that plasma rotation, plasma current and coupling to other modes have no strong impact on the rotation frequency of the mode. A proposed simple analytical model gives a good description of the experimental results (which uses cylindrical approximation and assumes the ideal character of the mode).

The RWM is a common problem for various toroidal devices (RFPs, Tokamaks). Thus, stabilization of RWMs has gained increasing attention in the fusion community during recent years. RFP experiments have demonstrated the control of multiple static RWMs with feedback coils $[12,13]$. The critical point for stabilization of the RWM in tokamaks is the prevention of the wall locking. When a mode locks, it grows rapidly and becomes strong and extremely difficult to stabilize with external coils (the number of which is also limited 
compared to RFPs). In this situation, the important task is the prevention of the mode locking and the fast unlocking of the mode from the wall. In this respect, RFPs are good test devices for testing different control schemes which may then be transferred to tokamaks. The presented results with externally induced rotation allow one to investigate the physical mechanism of the unlocking and induced rotation and thus can be considered as a first step for clarifying the physics of these processes. In some respects, our experiments are similar to the MHD spectroscopy in tokamaks [14] and may be used for future comparison. It is clear that the achievable rotation frequencies using external coils are of the order of the inverse resistive wall time, which is much slower compared to the plasma rotation frequency, but are of the same order as the rotation frequency of RWMs in tokamak. Thus, prevention of locking requires continuous control of the mode rotation, which is a challenging task. Further investigations are required to understand the feasibility and effectiveness of this approach.

An other interesting application of the rotation experiments is the investigation of the influence of the plasma rotation on the mode. Theoretical predictions show that plasma rotation is able to stabilize RWMs also in RFPs, but the rotation frequency should be very high (a few percent of the Alfvén velocity) [15]. Such rotation is not achievable in present RFPs where no sources of additional momentum input are available at present and the input power is mainly ohmic. It is interesting to note that initial predictions, based on a simple fluid model, give a small dissipation value also for tokamaks (and thus, only a small stabilizing effect from the plasma rotation on the mode), and that only the inclusion of kinetic effects substantially changes the dissipation value $[16,17]$. Thus, the experimental proof of the theory would be also interesting for any achievable frequency range.

The extension of similar experiments to other toroidal devices would also help to understand the role of passive and active boundary conditions in the determination of the equilibrium angular velocity for a given phase shift between plasma mode and the external perturbation

Acknowledgements: We would like to thank S. Günter for helpful discussions about RWM results and RWM physic and G. Marchiori and A. Soppelsa for help in running their model of the RFX-mod active control system and G.D. Conway for his remarks.

\section{References}

[1] P.R.Brunsell et. al., Phys. Rev. Lett., 93, 225001 (2004).

[2] R. Paccagnella et al., Phys. Rev. Lett., 97, 075001 (2006) 
[3] Bishop C.M., Plasma Phys. Controll. Fus. 31, 1179 (1989).

[4] Bolzonella T. et. al, Phys. Rev. Lett. 101, 165003 (2008)

[5] Igochine V. et. al., $35^{\text {th }}$ EPS conference on plasma physics, Crete 9-13 June 2008, P2.066

[6] Sonato P. et. al., Fusion Engineering and Design 66-68 (2003) 161-168

[7] G. Marchiori and A. Soppelsa, Fusion Eng. Des. 82, 1015 (2007).

[8] A. Bondeson et. al., Nucl. Fusion 42 (2002) 768-779

[9] R. Paccagnella, et. al., Nucl. Fusion 42 (2002) 1102-1109

[10] V.D. Pustovitov, Plasma Physics Reports, Vol. 27, No. 3, 2001, pp. 195-204

[11] Guo S. C. and Chu M. S., Phys. of Plasmas 8 (2001) 3342-3355

[12] Brunsell P.R. et. al., Phys. Rev. Lett., 93, 225001 (2004)

[13] Bolzonella T. et. al, Fusion Engineering and Design 82 (2007) 1064-1072

[14] Reimerdes H, et. al., Nucl. Fusion 45 (2005) 368-376

[15] S. C. Guo, J. P. Freidberg, and R. Nachtrieb, Phys. of Plasmas 6, 3868 (1999)

[16] Bondeson A. and Chu M. S., Phys. Plasmas 3 (8), August 1996, 3013

[17]Liu Y., et. al. , Nucl. Fusion 44 (2004) 232-242 